\title{
Treatment outcome of multidrug/extensively drug-resistant tuberculosis in Latvia, 2000- 2004
}

\author{
V. Leimane*, G. Dravniece*, V. Riekstina*, I. Sture*, S. Kammerer\#, M.P. Chen\#, \\ G. Skenders* and T.H. Holtz ${ }^{\#}$
}

ABSTRACT: In the present study, we characterised drug-resistance patterns, compared treatment outcome between extensively and nonextensively drug-resistant tuberculosis (nonXDR-TB) cases, and assessed risk factors for poor outcome in a high-prevalence country that screens all TB patients for first-line anti-TB drug resistance.

We reviewed drug susceptibility test results among all pulmonary TB cases in Latvia diagnosed from 2000-2004, as well as demographic and clinical characteristics, drug-resistance patterns, and treatment outcomes.

During the 5-yr period, 1,027 multidrug-resistant tuberculosis (MDR-TB) cases initiated treatment. Among all cases, the proportion that experienced an outcome of cure or completion increased from 66.2 to $70.2 \%$ ( $p=0.06$ for linear trend). Among the $48(4.7 \%)$ XDR-TB cases, 18 (38\%) were cured, four $(8 \%)$ died, three $(6 \%)$ defaulted, and treatment failed in $23(48 \%)$. In proportional-hazards analysis, characteristics significantly associated with poor outcome included XDR-TB, being retired, presence of bilateral cavitation, and previous MDR-TB treatment history for those aged $\geqslant 55$ yrs.

Overall, treatment success among all MDR-TB cases increased over time. Strategies to prevent transmission of XDR-TB and to further improve treatment outcome are crucial for the future of TB control in Latvia.

KEYWORDS: Multidrug-resistant tuberculosis, tuberculosis

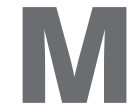
ultidrug-resistant tuberculosis (MDR$\mathrm{TB})$, caused by a strain resistant to at least isoniazid and rifampicin, the two most effective anti-TB first-line drugs (FLDs), poses a challenge to many national TB control programmes [1]. The proportion achieving cure and completion is lower, treatment duration is longer, and programmes must devote considerable human and financial resources to the administration of second-line drugs (SLDs). Extensively drugresistant (XDR)-TB, a subset of MDR-TB, has been defined as MDR-TB caused by a Mycobacterium tuberculosis isolate resistant to at least isoniazid, rifampicin, plus any fluoroquinolone (FQ), and at least one of three injectable SLDs (capreomycin, kanamycin or amikacin) $[2,3]$. XDR-TB magnifies the challenges in drug-resistant TB control, as it is especially lethal among HIV-infected persons [4]. However, the first reports of XDR-TB were in
non-HIV-infected individuals from Europe, demonstrating higher risk of death and a longer time required for culture conversion [5, 6]. Multiple convenience sample studies from Spain, Iran, Taiwan, and South Korea have been reported, which included treatment outcome data with subnational denominators [7-14]. Population-based MDR-TB and XDR-TB incidence data are now available from the United States [15], as well as from South Korea, Estonia, Peru and Tomsk (Russia), although the proportion of persons with TB infected with HIV in these locations was very low [12-14, 16-18]. National experience managing MDR-TB and XDR-TB cases over time from a highprevalence country is uncommon.

Latvia has one of the highest known prevalences of MDR-TB in the world, currently an estimated $14.3 \%$ among all reported TB cases [19]. Latvia
AFFILIATIONS

*State Agency of Tuberculosis and Lung Diseases, Riga, Latvia. \#Centers for Disease Control and Prevention, Atlanta, GA, USA.

CORRESPONDENCE

T.H. Holtz

Centers for Disease Control and

Prevention

1600 Clifton Road

MS E-45

Atlanta, GA 30333

USA

E-mail: tholtz@th.cdc.gov

Received:

Jan 072010

Accepted after revision:

Jan 152010

First published online:

Feb 252010 
introduced the World Health Organization (WHO)-recommended DOTS (directly observed treatment, short course) strategy in 1995 and a specialised component to diagnose and treat MDR-TB in 1998. Since that time, Latvia has been unusual amongst high-resistance prevalence countries for its policy of screening all TB patients for FLD resistance, which has been shown to improve outcome [12]. By systematically addressing programme deficiencies, Latvia reduced the number of newly registered MDR-TB cases by 63\%, from 332 cases in 1997 to 124 in 2007 [20]. Initially, the proportion achieving culture conversion and treatment success was encouraging, although lower among those co-infected with HIV [21-23]. As a proportion of newly diagnosed TB cases, MDR-TB declined from $12.2 \%$ in 1997 to $10.8 \%$ in 2008 [19]. MDR-TB cases resistant to a high number of SLDs, including those meeting the working definition of XDR-TB, have proven more difficult to treat $[24,25]$.

The ability to examine national longitudinal experience of MDR-TB management, and the emergence of XDR-TB as a potential threat, is rather unique among high MDR-TB prevalence countries [25]. The objectives of the present analysis are to provide a national population-based description of the treatment outcomes of MDR-TB and XDR-TB cases, to characterise drug-resistance patterns, and analyse risk factors for poor outcome under Latvia's MDR-TB treatment strategy between 2000-2004.

\section{METHODS}

\section{Cases and setting}

Our analysis included all new and previously treated pulmonary MDR-TB civilian and prison cases diagnosed and registered in the Latvian national TB registry during 20002004. All cases had sputum specimens submitted for $M$. tuberculosis culture and drug susceptibility testing at the time of diagnosis. The treatment cohort consisted of all patients who began individualised treatment for MDR-TB and XDR-TB with anti-TB SLDs between January 1, 2000 and December 31, 2004 at the State Agency of Tuberculosis and Lung Diseases (SATLD, Riga, Latvia).

\section{Definitions}

We regarded a person as an MDR-TB case if their initial isolate of $M$. tuberculosis showed in vitro resistance to at least isoniazid and rifampicin. Patients could be registered and initiate treatment for more than one treatment episode (defined as a case) during the analysis period. A case was regarded as meeting the XDR-TB definition if the initial MDR-TB isolate also showed resistance to any FQ and at least one of two injectable SLDs (capreomycin or kanamycin) tested in Latvia [26]. MDR-TB cases not meeting the XDR-TB definition are referred to as non-XDR-TB cases.

For this analysis we applied MDR-TB outcome definitions used by the WHO for MDR-TB cohort analysis [27]. We combined treatment cure and completion as treatment success, and death (from any cause) and failure as poor outcomes.

\section{Laboratory tests}

We conducted drug-susceptibility testing (DST) for FLDs on all diagnostic TB cultures. SLD DST was begun on all MDR-TB cases starting in 2000. All mycobacterium cultures were performed according to international standards at the Latvian national TB reference laboratory in Riga using conventional Lowenstein-Jensen solid media or the BACTEC rapid growth method [28]. DST performed on sputum $M$. tuberculosis isolates by the national laboratory was done with the absolute concentration method and Lowenstein-Jensen media, and followed accepted international practice [29].

\section{Treatment}

Regimens were individually tailored according to the drugsusceptibility profile of the cases' initial MDR-TB isolate. Treatment with SLDs was started as soon as resistance to isoniazid and rifampicin was documented. Empirical regimens for MDR-TB and XDR-TB including SLDs were used (based on MDR-TB case contact's DST and SLDs that had not been used previously) until the results of SLD (and final FLD) DST were received. Drugs used for MDR-TB treatment included ethambutol, pyrazinamide, ofloxacin, kanamycin, capreomycin, prothionamide, cycloserine, para-aminosalicylic acid (PAS), and thiacetazone. The initial empirical regimen normally consisted of between four and eight drugs (including one injectable) and a FQ. To strengthen regimens containing only three SLDs in patients with extensive resistance profiles, the third-line drugs amoxicillin, clavulanate and clarithromycin were used.

Drugs were given daily by direct observation for the duration of treatment. After sputum culture conversion, the injectable drug was continued five times per week for 4-6 months (4 months after culture conversion).

\section{Data collection}

We used data from hospital and ambulatory records, and bacteriological laboratory reports that were entered into an MDR-TB database designed for the Latvian programme. Available variables have been described elsewhere [21]

\section{Statistical analysis}

We described demographic, clinical and treatment characteristics and compared differences between MDR-TB and XDRTB cases, utilising the Chi-squared test for dichotomous variables, paired t-test for normally distributed continuous variables, and the Wilcoxon rank sum test for non-normally distributed continuous variables. We used Chi-squared test for trend in stratified analysis, and slope for linear trend in the regression model of longitudinal data. A $p<0.05$ was considered statistically significant. We calculated univariate risk ratios (RR) to determine the risk of poor outcome for nondefaulting MDR-TB cases. To determine the significance when comparing drug-resistance groups with outcome, we used the log-rank test of the Kaplan-Meier survival curves.

Covariates with clinical relevance for poor outcome were retained for multivariable Cox proportional hazards analysis. Cases were censored if they did not reach a poor treatment outcome of death or failure at the end of the cohort period. Data were analysed using Epi Info Version 6.04d (Epi Info, Atlanta, GA, USA) and SAS for Windows Release 9.1 (SAS Institute, Cary, NC, USA). Missing data were not replaced or imputed. Additional detail on methodology is provided in the online data supplement. 
This analysis was determined to be a programmatic evaluation and approved for non-human subject research by the Office of the Associate Director for Science, National Center for HIV, Viral Hepatitis, STD, and TB Prevention, US Centers for Disease Control and Prevention (CDC, Atlanta, GA, USA). The protocol was approved by the SATLD Medical Ethics Committee. Confidentiality protections were assured for all persons in the study.

\section{RESULTS \\ MDR-TB and XDR-TB population-based registration, 2000- 2004}

The final 2000-2004 treatment cohort contained 1,027 MDR-TB case treatment episodes (received by 996 persons) who initiated therapy with SLDs (table 1). 48 (4.7\%) cases met the XDR-TB definition (among 47 persons), and 979 cases were non-XDR-TB cases.

\section{Non-XDR-TB and XDR-TB demographic and clinical characteristics}

Males with non-XDR-TB had a median age of $43.1 \mathrm{yrs}$, and those with XDR-TB had a median age of 37.4 yrs $(p=0.12)$ (table 2). There were no statistically significant demographic differences between the groups except reported contact



history: 169 out of 969 (17.3\%) non-XDR-TB cases versus 15 out of $48(31.2 \%)$ XDR-TB cases reported contact with an MDRTB case $(p=0.02)$. XDR-TB and non-XDR-TB cases differed in past TB treatment history. Among both groups, 379 (36.9\%) reported never receiving treatment for TB previously. 16 out of $48(33.3 \%)$ XDR-TB cases were previously treated for MDR-TB, versus 100 out of $979(10.2 \%)$ of non-XDR-TB ( $p=0.0001)$. Three of $48(6.2 \%)$ XDR-TB cases were HIV-infected, versus 29 out of $974(3.0 \%)$ of non-XDR-TB $(p=0.2)$. Non-XDR-TB cases were more likely to have existing comorbidities (453 out of 979 $(46.3 \%)$ versus $15 / 48(31.3 \%) ; p=0.04)$ such as diabetes, hepatitis, or cardiovascular disease.

Initial M. tuberculosis isolates from the 48 XDR-TB cases were resistant to a median of eight drugs (interquartile range (IQR 7-9)), versus five drugs (IQR 4-7; $<<0.001$ ) for the non-XDR-TB cases. Resistance to kanamycin was more common than resistance to capreomycin in both groups. XDR-TB cases were statistically more likely to be additionally resistant to kanamycin, capreomycin, prothionamide, ethambutol and pyrazinamide (table 3). Among all 1,027 MDR-TB cases, 469 (45.6\%) were resistant to all five FLDs (isoniazid, rifampicin, ethambutol, pyrazinamide, streptomycin). $50 \%$ of cases were resistant to either kanamycin or capreomycin, and 178 $(17.3 \%)$ were resistant to both.

\section{Treatment characteristics of non-XDR-TB and XDR-TB cases}

The median total duration of treatment for all 1,027 MDR-TB cases was 550 days (IQR 366-642 days). There was no statistically significant difference in treatment duration between non-XDR-TB and XDR-TB cases. XDR-TB cases did not die more quickly (median 113 days versus 183 days; $\mathrm{p}=0.36$ ) (table 4). Treatment for XDR-TB cases utilised a median of six drugs (IQR 5-7) for $\geqslant 3$ months; however, the case's isolate showed susceptibility to only two of the drugs used (IQR 0-4). By comparison, non-XDR-TB cases were treated with a median of six drugs (IQR 5-6; $\mathrm{p}=0.16$ ) with susceptibility to four drugs (IQR 3-5; $p=0.005$ ).

Among all cases the most commonly used injectable was kanamycin $(50.8 \%)$, followed by capreomycin (47.3\%). 138 $(13.4 \%)$ cases received an injectable medication for $<3$ months; 79 of these went on to cure or completion. The SLDs ofloxacin $(n=982,95.6 \%)$, cycloserine $(n=902,87.8 \%)$, prothionamide $(n=791,77.0 \%)$, thiacetazone $(n=680,66.2 \%)$, and PAS $(n=558$, $54.3 \%$ ) were all used in a majority of cases. Amoxicillin/ clavulanate $(n=133,13.0 \%)$ and clarithromycin $(n=55,5.4 \%)$ were infrequently used, and moxifloxacin was used in only five XDR-TB cases and one non-XDR-TB case in late 2004. For 73 non-XDR-TB cases and six XDR-TB cases, surgical procedures were used during treatment: wedge resection (24 cases), polysegmental resection (23 cases), lobectomy (15 cases), pneumonectomy (10 cases), pleuro-pneumonectomy (seven cases), or other (six cases).

Among non-XDR-TB and XDR-TB cases, 208 (21.2\%) and three $(6.3 \%)$, respectively, culture converted on FLDs (before DST laboratory confirmation of MDR-TB) prior to starting SLDs. Among those with a positive culture taken at initiation of MDRTB treatment, there was no statistically significant difference in the time to culture conversion between non-XDR-TB and XDR-TB cases (combined median 62 days, IQR 34-109 days), 
TABLE 2 Demographic and clinical characteristics of 1,027 multidrug-resistant tuberculosis (MDR-TB) cases, stratified by nonextensively drug-resistant (XDR)-TB and XDR-TB status, Latvia, 2000-2004\#

\begin{tabular}{|c|c|c|c|}
\hline & Non-XDR-TB & XDR-TB & p-value \\
\hline Cases n & 979 & 48 & \\
\hline \multicolumn{4}{|c|}{ Demographic characteristic } \\
\hline \multicolumn{4}{|l|}{ Sex } \\
\hline Male & 746 (76.2) & $34(70.8)$ & 0.40 \\
\hline Female & $233(23.8)$ & $14(29.2)$ & Ref \\
\hline $18-24$ & $66(6.7)$ & $6(12.5)$ & \\
\hline $25-34$ & $206(21.0)$ & $8(16.7)$ & \\
\hline $35-44$ & $284(29.0)$ & $17(35.4)$ & \\
\hline $45-54$ & $240(24.5)$ & $13(27.0)$ & \\
\hline$>55$ & $176(18.0)$ & $3(6.3)$ & \\
\hline \multicolumn{4}{|l|}{ Age yrs } \\
\hline Riga, Latvia & $333(34.0)$ & $22(45.8)$ & 0.09 \\
\hline Other & $646(66.0)$ & $26(54.2)$ & Ref \\
\hline \multicolumn{4}{|l|}{ Birthplace } \\
\hline Latvia & $859(87.7)$ & 40 (83.3) & 0.37 \\
\hline Other & $120(12.3)$ & $8(16.7)$ & Ref \\
\hline \multicolumn{4}{|l|}{ Housing } \\
\hline Tenant & $212(21.6)$ & $13(27.1)$ & 0.37 \\
\hline Owner & $767(78.4)$ & 35 (72.9) & Ref \\
\hline \multicolumn{4}{|l|}{ Employment } \\
\hline Student/retired & $270(27.6)$ & $18(37.5)$ & 0.14 \\
\hline Unemployed & $547(55.9)$ & $26(54.2)$ & 0.81 \\
\hline Employed & $162(16.5)$ & $4(8.3)$ & Ref \\
\hline Any & $313(32.0)$ & $18(37.5)$ & Ref \\
\hline Unknown & $1(0.1)$ & & \\
\hline \multicolumn{4}{|c|}{ Past/current heavy alcohol use } \\
\hline None & $432(44.1)$ & $17(35.4)$ & 0.23 \\
\hline Ever & $547(55.9)$ & $31(64.6)$ & Ref \\
\hline \multicolumn{4}{|c|}{ Injection drug use ever } \\
\hline No & $907(92.6)$ & $43(89.5)$ & 0.58 \\
\hline Yes & $45(4.6)$ & $3(6.3)$ & Ref \\
\hline Unknown & $27(2.8)$ & $2(4.1)$ & \\
\hline \multicolumn{4}{|c|}{ Started treatment while incarcerated } \\
\hline No & $904(92.3)$ & $45(93.8)$ & 0.72 \\
\hline Yes & $75(7.7)$ & $3(6.2)$ & Ref \\
\hline \multicolumn{4}{|l|}{ Clinical characteristics } \\
\hline \multicolumn{4}{|l|}{$\mathrm{BMI} \mathrm{kg} \cdot \mathrm{m}^{-2}$} \\
\hline Males & $20.6(19.0-22.3)$ & $21.1(19.3-22.2)$ & $0.42^{5}$ \\
\hline Females & $20.3(18.6-22.5)$ & $19.7(17.2-22.4)$ & $0.41^{\S}$ \\
\hline Males $<18.5^{\circ}$ & 137/728 (18.8) & $7 / 34(20.6)$ & 0.79 \\
\hline Females $<18.5^{+}$ & 54/228 (23.6) & $4 / 14(28.6)$ & 0.67 \\
\hline \multicolumn{4}{|l|}{ HIV status } \\
\hline Infected & $29(3.0)$ & $3(6.2)$ & 0.2 \\
\hline
\end{tabular}




\begin{tabular}{|c|c|c|c|}
\hline & Non-XDR-TB & XDR-TB & p-value \\
\hline Unknown & $5(0.5)$ & $0(0)$ & \\
\hline \multicolumn{4}{|l|}{ Category of MDR-TB } \\
\hline Never treated for TB & $359(36.7)$ & $20(41.7)$ & Ref \\
\hline \multicolumn{4}{|l|}{ Site of MDR-TB } \\
\hline Pulmonary and extrapulmonary & $57(5.8)$ & $0(0)$ & 0.08 \\
\hline Pulmonary & $970(94.2)$ & $48(100)$ & Ref \\
\hline \multicolumn{4}{|l|}{ Radiological findings at onset } \\
\hline Bilateral cavitary & $208(21.3)$ & $15(31.3)$ & 0.10 \\
\hline \multicolumn{4}{|l|}{ Colony count of initial culture among positive } \\
\hline $3-4$ & $413(54.1)$ & $28(65.1)$ & 0.16 \\
\hline $1-2$ & $351(45.9)$ & $15(34.9)$ & Ref \\
\hline $\begin{array}{l}\text { Drugs to which } M \text {. tuberculosis resistant } \\
\text { at treatment onset } \\
\text { at treatment initiation }\end{array}$ & $5(4-7)$ & $8(7-9)$ & $<0.001^{\mathrm{s}}$ \\
\hline $2-4$ & $285(29.1)$ & & \\
\hline 5 & $229(23.4)$ & & \\
\hline 6 & $186(19.0)$ & $6(12.5)$ & \\
\hline 7 & $127(13.0)$ & $13(27.1)$ & \\
\hline 8 & $103(10.5)$ & $10(20.8)$ & \\
\hline
\end{tabular}

Data are presented as $\mathrm{n}(\%)$ or median (interquartile range), unless otherwise stated. M. tuberculosis: Mycobacterium tuberculosis; Ref: reference. ${ }^{\#}$ : year 2000 for

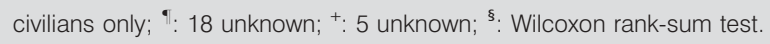

although more XDR-TB cases than non-XDR-TB cases never experienced culture conversion $(57.8 \%$ versus $19.8 \%$; $<<0.001)$.

Treatment adherence was similar between the two groups; $46 \%$ in both groups had one interruption of $\geqslant 5$ weeks. More than three-quarters of all MDR-TB cases experienced adverse events. The most common adverse events were nausea, vomiting, diarrhoea and abdominal pain. No XDR-TB cases discontinued treatment because of adverse events.

\section{Treatment outcome, 2000-2004}

Among the entire MDR-TB cohort, 697 (67.9\%) cases experienced successful treatment outcome (table 5), which increased from $66.2 \%$ in 2000 to $70.2 \%$ in 2004 (fig. 1). Outcome for nonXDR-TB cases is shown in table 6. Treatment success among previously treated cases improved from $62.4 \%$ in 2000 to $66.0 \%$ in 2004 (table 6). Among the 48 XDR-TB cases, 18 (38\%) were cured or completed therapy, $4(8 \%)$ died, 3 (6\%) defaulted, and treatment failed in $23(48 \%)$. HIV infection was not significantly associated with XDR-TB (RR 1.0; $\mathrm{p}=0.20)$ nor poor outcome among either non-XDR-TB cases (RR 1.3; $\mathrm{p}=0.19)$ or all MDR-TB cases (RR 1.4; $\mathrm{p}=0.15$ ).
Our univariate analysis for risk factors associated with poor outcome among all MDR-TB cases found that past history of TB treatment, and the severity of current disease (bilateral cavitation, culture colony count), were significantly related to poor outcome (see table 1 of the online supplementary material). Males had a higher risk than females. A history of incarceration and present or past heavy alcohol use were significantly associated with poor outcome. Resistance to seven or more drugs, and using less than four drugs to which the isolate was susceptible, were both independently and significantly related to poor outcome. Converting to culturenegative after 62 days versus before 62 days was significantly related to a poor treatment outcome (36.8 versus $6.2 \%$; $\mathrm{p}<0.001)$. Surgery was not significantly associated with poor outcome. In univariate proportional-hazards modelling the addition of one drug to which the case's isolate was initially susceptible decreased the hazard of poor outcome by $33 \%$ (hazard ratio (HR) $0.67,95 \%$ CI $0.60-0.75 ; \mathrm{p}<0.0001$ ).

Figure 2 shows the Kaplan-Meier survival distribution estimates of survival to poor outcome among four groups of MDRTB. Cases of XDR-TB (4.7\%) reached a poor outcome much more rapidly than MDR-TB cases resistant to a FQ but not an 


\begin{tabular}{|c|c|c|c|c|c|}
\hline \multirow{2}{*}{$\begin{array}{l}\text { TABLE } 3 \\
\text { Resistance }\end{array}$} & \multicolumn{5}{|c|}{$\begin{array}{l}\text { Resistance to anti-tuberculosis (TB) agents at the } \\
\text { initiation of treatment: 1,027 multidrug-resistant } \\
\text { (MDR)-TB cases stratified by non-extensively } \\
\text { drug-resistant (XDR)-TB and XDR-TB status, } \\
\text { Latvia, 2000-2004 }\end{array}$} \\
\hline & & Non-XDR-TB & XDR-TB & $p$-value & Total \\
\hline Cases $\mathrm{n}$ & & 979 & 48 & & 1027 \\
\hline \multicolumn{6}{|l|}{ First-line drugs } \\
\hline Streptomycin & & 929 (94.9) & 47 (97.9) & 0.34 & $976(95.0)$ \\
\hline Pyrazinamide & & $662(67.6)$ & 43 (89.6) & 0.001 & $705(68.7)$ \\
\hline Ethambutol & & $596(60.9)$ & $38(79.2)$ & 0.01 & $634(61.7)$ \\
\hline \multicolumn{6}{|c|}{ Second-line drugs } \\
\hline Kanamycin & & $396(40.5)$ & $41(85.4)$ & $<0.001$ & $437(42.6)$ \\
\hline Capreomycir & & $223(22.8)$ & 27 (56.3) & $<0.001$ & $250(24.3)$ \\
\hline Ofloxacin & & $18(1.8)$ & $48(100)$ & $<0.001$ & $66(6.4)$ \\
\hline Prothionamic & & $238(24.3)$ & $24(50.0)$ & $<0.001$ & $262(25.5)$ \\
\hline PAS & & $267(27.3)$ & $14(29.2)$ & 0.77 & $281(27.4)$ \\
\hline Thiacetazone & & $130(13.3)$ & $9(18.8)$ & 0.27 & $139(13.5)$ \\
\hline Cycloserine & & $18(1.8)$ & $2(4.2)$ & 0.25 & $20(2.0)$ \\
\hline
\end{tabular}

Data are presented as $\mathrm{n}$ or $\mathrm{n}(\%)$. PAS: para-aminosalicylic acid. ${ }^{*}$ : year 2000 for civilians only. injectable $(1.8 \%)$, as well as MDR-TB cases resistant to an injectable but not a FQ $(44.8 \%)$, versus all other MDR-TB cases (log rank test $\mathrm{p}<0.0001)$.

In multivariate time-dependent Cox proportional hazards regression, factors independently associated with poor outcome included the presence of XDR-TB resistance on the initial isolate (HR 1.96, 95\% CI 1.14-3.36; $\mathrm{p}=0.02)$, having bilateral cavitation on initial radiography (HR 1.90, 95\% CI 1.19-3.03; $\mathrm{p}=0.007$ ), and being retired or a student (HR 2.56, 95\% CI 1.28$5.12 ; \mathrm{p}=0.008$ ) (table 7). The impact of XDR-TB on poor outcome did not change over time $(p=0.47)$. Several interaction terms were found: cases $>55$ yrs of age were more than four times more likely to have a poor outcome if they had been previously treated for MDR-TB (HR 4.34, 95\% CI 1.62-11.6; $\mathrm{p}=0.003)$, and cases who converted their sputum culture to negative after 62 days were nearly four times more likely to have a poor outcome if their total treatment time exceeded 100 days (HR 3.83, 95\% CI 1.81-8.12; $\mathrm{p}=0.0005$ ).

\section{DISCUSSION}

The WHO estimates the incidence of MDR-TB to be $300,000-$ 600,000 cases annually, and prevalence three-fold higher, largely in low- and middle-income countries [30]. The global burden of XDR-TB is not currently known, as data are scarce. Latvia ranks

TABLE 4 Treatment characteristics of 1,027 multidrug-resistant tuberculosis (MDR-TB) cases, stratified by non-extensively drugresistant (XDR)-TB and XDR-TB status, Latvia, 2000-2004 ${ }^{\#}$

\begin{tabular}{|c|c|c|c|}
\hline Cases n & 979 & 48 & \\
\hline \multicolumn{4}{|l|}{ Duration of therapy days } \\
\hline Cure & $599(538-671)$ & $641(547-857)$ & $0.10^{+}$ \\
\hline Completion & $618(386-665)$ & & \\
\hline Failure & $348(227-502)$ & $378(316-600)$ & $0.13^{+}$ \\
\hline Total cohort & $552(376-640)$ & $525(323-680)$ & $0.79^{+}$ \\
\hline Number of resistant drugs used for $\geqslant 3$ months & $<1(0-1)$ & $2(1-2)$ & $<0.001^{+}$ \\
\hline \multicolumn{4}{|l|}{ Culture conversion } \\
\hline Started therapy culture-negative & $208(21.2)$ & $3(6.3)$ & Ref \\
\hline For $\geqslant 5$ weeks & $435(44.4)$ & $23(47.9)$ & 0.63 \\
\hline For $<5$ weeks & $544(55.6)$ & $25(52.1)$ & Ref \\
\hline \multicolumn{4}{|l|}{ Missing $\geqslant 40$ doses } \\
\hline Yes & $368(37.6)$ & $16(33.3)$ & 0.55 \\
\hline No & $611(62.4)$ & $32(66.7)$ & Ref \\
\hline \multicolumn{4}{|l|}{ Adverse events during treatment } \\
\hline Any & $759(77.5)$ & $39(81.2)$ & 0.55 \\
\hline None & $220(22.5)$ & $9(18.8)$ & Ref \\
\hline Adverse events reported per person & $3(1-6)$ & $4(1-6)$ & $0.53^{+}$ \\
\hline
\end{tabular}

Data are presented as $n$ (\%) or median (interquartile range), unless otherwise stated. Ref: reference. ${ }^{\#}$ : year 2000 for civilians only; ${ }^{\circ}$ : data are presented as mean (95\% $\mathrm{Cl}){ }^{+}$: Wilcoxon rank-sum test. 


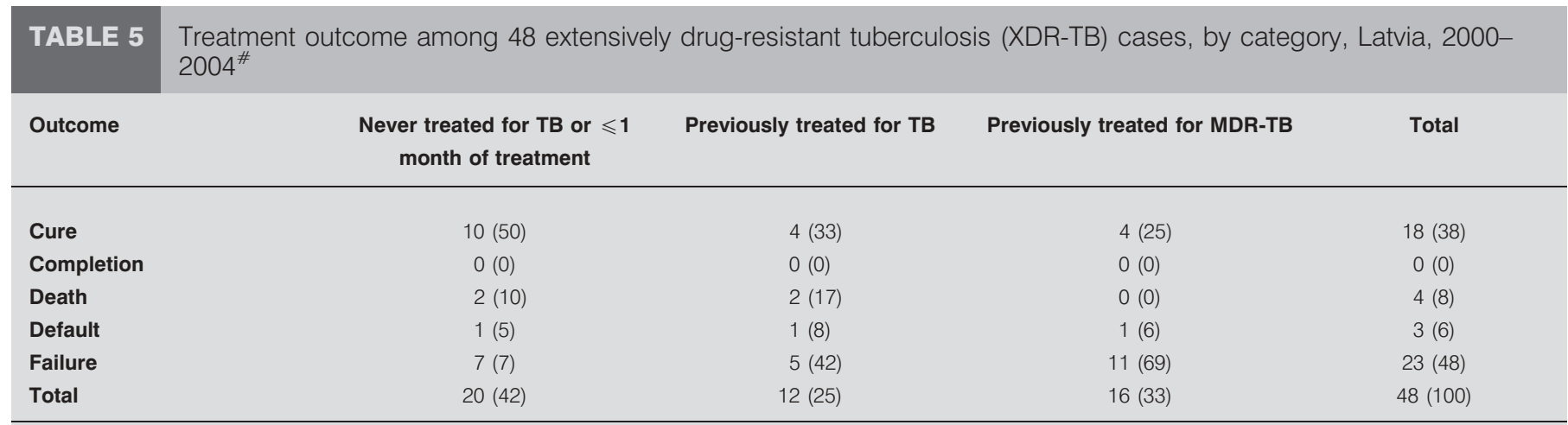

Data are presented as $n$ (\%). MDR-TB: multidrug-resistant TB. ${ }^{\#}$ : year 2000 for civilians only.

26th among WHO's 27 high-MDR-TB burden countries. Our data show that $\sim 5 \%$ of MDR-TB cases treated in Latvia have XDR-TB. A similar proportion is reported from neighboring Estonia and other former Soviet Union regions [18, 25].

We found treatment outcome to be significantly associated with previous treatment history. Latvia's sustained overall proportion achieving cure and completion near $70 \%$ over 5 yrs
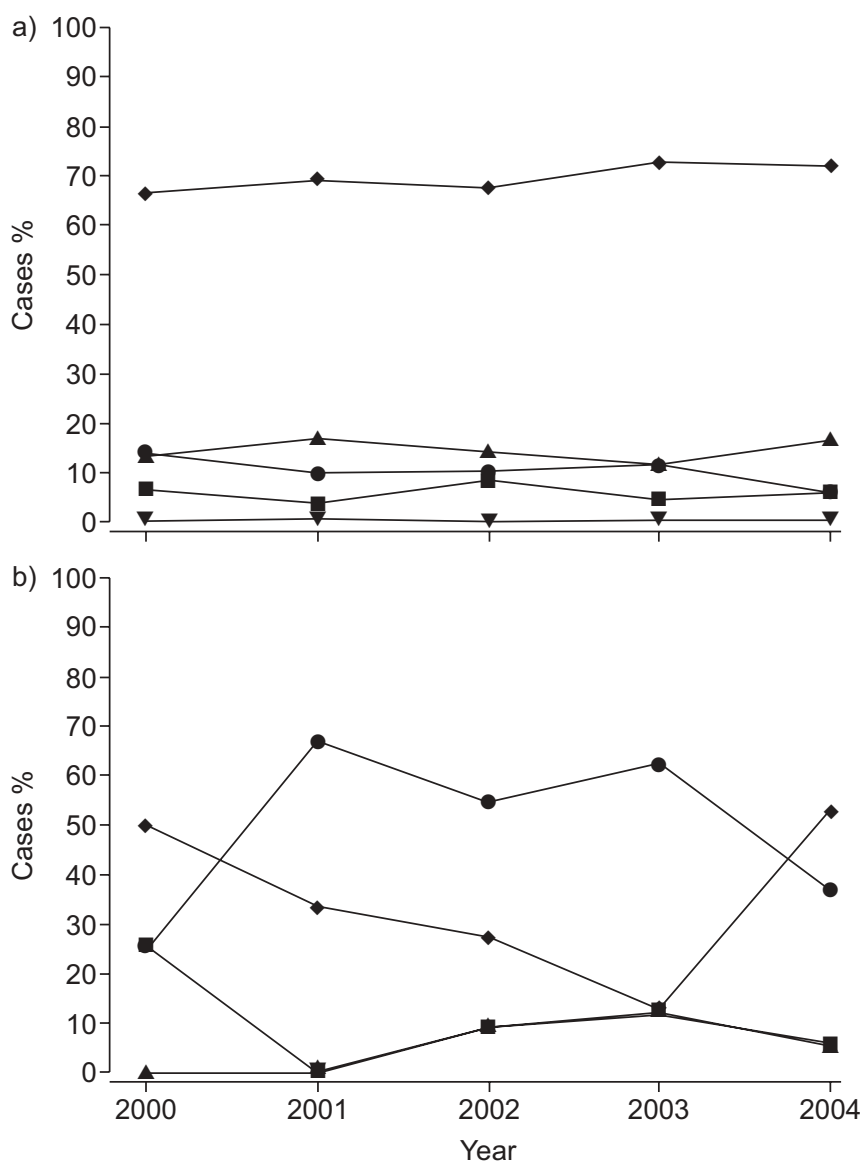

FIGURE 1. Treatment outcome over time in a) non-extensively drug-resistant tuberculosis (non-XDR-TB) and b) XDR-TB cases, Latvia, 2000-2004. $\bullet$ : success; is excellent, with improvements over time largely due to higher proportions achieving cure and completion among cases previously treated for TB and MDR-TB. That, combined with decreasing numbers of re-treatment cases, resulted in better programme performance. However, a lower proportion achieving cure $(38 \%)$ in patients resistant to critical bactericidal SLDs (XDR-TB) poses challenges for a national programme burdened with high levels of resistance and a limited budget. Latvia has shown that XDR-TB can be treated without sacrificing overall MDR-TB program effectiveness. Optimally, studies have shown the proportion of XDR-TB achieving cure and completion $>50 \%$ in sub-national populations $[17,18]$.

What has led to the emergence of XDR-TB in Latvia? In most cases, basic TB control practices are suboptimal in areas where MDR-TB has emerged. But this has not been the recent case in Latvia, since DOTS was implemented on a nationwide scale in 1995. Low cure and case detection rates have improved greatly, such that they are now comparable to those of western Europe [1]. In addition, the factors that fostered the creation of MDRTB in the 1990s are no longer present in Latvia, such as inadequate regimens for $\mathrm{TB}$, acute drug shortages, inadequately controlled administration of FLDs, and failure of treatment with first-line regimens, although they may be present in other countries in the former Soviet Union. The acquisition of resistance during prior treatment with SLDs, and nosocomial transmission, may have played a role [25].

A history of previous MDR-TB treatment was three times more common in the XDR-TB patient population than the non-XDRTB population, which is likely to be due to the acquisition of resistance over time. However, roughly the same proportion had never been treated for TB in both groups. Transmission of resistant MDR-TB and XDR-TB organisms (primary drug resistance) occurred. 20 of $48 \mathrm{XDR}-\mathrm{TB}$ cases had never received TB treatment, and only three $(15 \%)$ of those had been incarcerated (for an unknown length of time). In addition, 12 XDR-TB cases had previously received only FLDs, and were likely primary XDR-TB cases. The prevalence of resistance due to the transmission of already-resistant strains is unlikely to be reduced by the implementation of a well-run DOTS programme [31]. Implementation of rapid molecular diagnostic tests would have detected these cases earlier and led to earlier initiation of treatment [32]. The availability of treatment at earlier diagnosis will eventually reduce transmission. Now 
TABLE 6 Treatment outcome among 979 non-extensively drug-resistant tuberculosis (TB) cases by category, Latvia, 2000-2004

\begin{tabular}{lcccc} 
Outcome & $\begin{array}{c}\text { Never treated for TB or } \leqslant \mathbf{1} \\
\text { month of treatment }\end{array}$ & Previously treated for TB & Previously treated for MDR-TB & Total \\
\hline Cure & $271(75.5)$ & $344(66.2)$ & $48(48.0)$ & $0(0)$ \\
Completion & $10(2.8)$ & $6(1.1)$ & $10(10.0)$ & $16(1.6)$ \\
Death & $15(4.2)$ & $31(6.0)$ & $15(15.0)$ & $56(5.7)$ \\
Default & $46(12.8)$ & $81(15.6)$ & $27(27.0)$ & $142(14.5)$ \\
Failure & $16(4.4)$ & $58(11.1)$ & $100(10.2)$ & $101(10.3)$ \\
On treatment & $1(0.3)$ & $520(53.1)$ & $979(100)$ \\
Total & $359(36.7)$ & & \\
\hline
\end{tabular}

Data are presented as n (\%). MDR-TB: multidrug-resistant TB. ${ }^{\#}$ : year 2000 for civilians only.

that line probe assays are being used in Latvia, further reduction of MDR-TB is expected.

Not surprisingly, we found that XDR-TB patient $M$. tuberculosis isolates are resistant to 2.5 drugs more than non-XDR-TB patient isolates. Resistance to other SLDs was extensive, with two patient isolates resistant to all drugs tested. The presence of XDR-TB clearly limits the ability to find a drug regimen to which the patient's isolate will be susceptible. However, if a patient's isolate is resistant to only the four most critical bactericidal drugs (isoniazid, rifampicin, a fluoroquinolone, and an injectable) for $\mathrm{TB}$, it may still be possible to use other FLD and an injectable that shows activity in vitro $[33,34]$. The newly released WHO Guidelines for the treatment of MDR-TB specifically call for the use of a minimum of four effective drugs to treat drug-resistant TB [27]. Our data demonstrate the validity of this recommendation.

We found that the time to culture conversion in both groups was not significantly different. However, many non-XDR-TB patients culture-converted to negative while on FLDs before starting MDR-TB treatment, which would have left patients

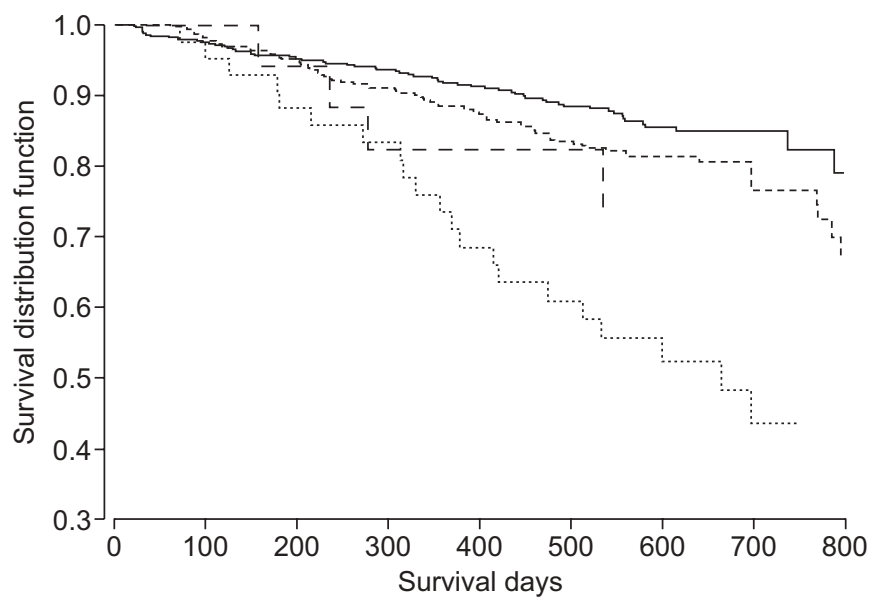

FIGURE 2. Kaplan-Meier estimates of survival to successful outcome among all multidrug-resistant tuberculosis cases stratified by resistance to any injectable but not a fluoroquinolone (---), a fluoroquinolone but not an injectable (-- -), both an injectable and a fluoroquinolone (extensively drug-resistant tuberculosis) $(\cdots \cdots \cdots . .$.$) , and all others (-)$ with higher resistance to convert using SLDs. In the era before rapid tests were being routinely used, many patients were put on FLDs when in fact they had MDR-TB. Conversion on FLDs could be due to sterilisation of parenchymal TB without killing of cavitary bacilli. However, three times more XDR-TB patients did not culture-convert at all, perhaps due to the higher mean number of drugs to which this group was resistant. For all patients in our model, conversion after 2 months of treatment was associated with a high hazard for poor outcome.

Drug susceptibility testing in Latvia is well-utilised, qualityassured, and reliable [35]. Every person with TB in Latvia receives at least one drug susceptibility test against FLDs at the beginning of treatment, one of the key aspects of management in our context that has allowed rapid diagnosis of MDR-TB (now performed in liquid media). This is a standard that should now apply to all regions with high levels of drug resistance like countries of the former Soviet Union. In particular, all TB isolates showing resistance to rifampicin should have SLD susceptibility testing performed according to international recommendations and standards [36, 37].

A key to long-term control of MDR-TB and XDR-TB is rapid identification of rifampicin resistance, improved laboratory infrastructure, prompt initiation of effective treatment regimens, and proper management of side-effects to avoid problems with nonadherence and prevent further amplification of resistance and creation of more MDR-TB and XDR-TB strains [38]. Patients in this region also frequently struggle with homelessness, alcoholism and poverty. Treatment in this population needs to be comprehensive, addressing all their social needs. Expansion of MDR-TB and XDR-TB treatment in Eastern European region is needed, where $53 \%$ of the projected number of MDR-TB cases worldwide will be treated, in order to prevent the further development of drug resistance to second-line injectables and FQs. Molecular epidemiology studies to determine the MDR-TB strains circulating in the community, in addition to the investigation and determination of the epidemiologic links between cases, are urgently needed [39].

The present study had several limitations. Although concordance between the Latvian national laboratory and its supranational laboratory for FLD and ofloxacin DST is high, we cannnot exclude the possibility of misclassification of drug-susceptibility test results of SLDs used for treatment. During the cohort 


\begin{tabular}{|c|c|c|c|}
\hline \multirow{2}{*}{$\begin{array}{l}\text { TABLE } 7 \\
\text { Characteris }\end{array}$} & \multicolumn{3}{|c|}{$\begin{array}{l}\text { Time-dependent Cox proportional hazards } \\
\text { multivariable model for poor outcome among } \\
994 \text { multidrug-resistant tuberculosis (MDR-TB) } \\
\text { patients, Latvia, 2000-2004 }\end{array}$} \\
\hline & & Hazard ratio $(95 \% \mathrm{Cl})$ & p-value \\
\hline \multicolumn{4}{|l|}{ Sex } \\
\hline Female & & 1.0 & \\
\hline Male & & $1.10(0.73-1.65)$ & 0.64 \\
\hline \multicolumn{4}{|l|}{ Employment } \\
\hline Employed & & 1.0 & \\
\hline Unemploye & & $1.78(0.91-3.48)$ & 0.09 \\
\hline Retired/stuc & & $2.56(1.28-5.12)$ & 0.008 \\
\hline \multicolumn{4}{|c|}{ Category of MDR-TB } \\
\hline Never treate & $d$ for TB & 1.0 & \\
\hline \multicolumn{4}{|c|}{ Age previously treated for TB yrs } \\
\hline$<35$ & & $1.27(0.71-2.27)$ & 0.41 \\
\hline $35-54$ & & $1.14(0.75-1.75)$ & 0.54 \\
\hline$\geqslant 55$ & & $1.67(0.97-2.88)$ & 0.07 \\
\hline \multicolumn{4}{|c|}{ Age previously treated for MDR-TB yrs } \\
\hline$<35$ & & $1.55(0.67-3.59)$ & 0.31 \\
\hline $35-54$ & & $1.66(0.94-2.94)$ & 0.08 \\
\hline$\geqslant 55$ & & $4.34(1.62-11.6)$ & 0.003 \\
\hline \multicolumn{4}{|c|}{ Cavitation on radiography } \\
\hline No cavitatio & & 1.0 & \\
\hline Unilateral ce & vitation & $1.22(0.78-1.92)$ & 0.39 \\
\hline Bilateral ca & tation & $1.90(1.19-3.03)$ & 0.007 \\
\hline \multicolumn{4}{|c|}{ Presence of XDR-TB in initial isolate } \\
\hline No & & 1.0 & \\
\hline Yes & & $1.96(1.14-3.36)$ & 0.02 \\
\hline \multicolumn{4}{|c|}{ Culture conversion } \\
\hline Began treat & nent culture-negative & 1.0 & \\
\hline \multicolumn{4}{|c|}{ Conversion before 62 days of treatment } \\
\hline Treatmen & time $\leqslant 100$ days & $0.88(0.25-3.03)$ & 0.84 \\
\hline Treatmen & time $>100$ days & $0.51(0.20-1.29)$ & 0.16 \\
\hline \multicolumn{4}{|c|}{ Conversion after 62 days of treatment } \\
\hline Treatmen & time $\leqslant 100$ days & $0.75(0.23-2.41)$ & 0.63 \\
\hline Treatmen & time $>100$ days & $3.83(1.81-8.12)$ & 0.0005 \\
\hline \multicolumn{4}{|c|}{ Drugs used in regimen for $\geqslant 3$ months } \\
\hline Kanamycin & & $0.60(0.43-0.83)$ & 0.002 \\
\hline Ofloxacin & & $0.54(0.30-0.97)$ & 0.04 \\
\hline Prothionam & & $0.64(0.45-0.90)$ & 0.01 \\
\hline Cycloserine & & $0.40(0.25-0.64)$ & $<0.001$ \\
\hline
\end{tabular}

period, amikacin was not available in Latvia and thus it was not included in the DST panel, nor was moxifloxacin or levofloxacin. The chances that a kanamycin-susceptible, amikacinresistant strain was misdiagnosed are extremely low. There is a lack of international standards for quality assurance methods for SLD testing and limited reproducibility of drug susceptibility testing for certain drugs, although drug susceptibility testing for aminoglycosides and FQs is reliable [40, 41]. WHO recommends that all three second-line injectables and at least one FQ are included in the second-line DST.

In summary, the Latvian programme has demonstrated that a high level of successful treatment outcome in persons with
MDR-TB can be sustained over time, but must be accompanied by SLD availability and quality-assured DST. The presence of XDR-TB is a risk factor for poor outcome, together with older age, low income, severity of disease, late culture conversion, and the use of less than 4 effective drugs in the regimen. Importantly, the implementation of rapid methods of early detection of drug-resistant TB in persons with TB diagnosed in countries with limited resources is urgently needed to avoid the further generation and transmission of XDR-TB and to improve the proportion achieving cure for persons with MDR-TB [42].

\section{SUPPORT STATEMENT}

Funding for this study was provided by the Latvian Ministry of Health. A small amount was contributed by the US Agency for International Development to support technical assistance to the project, but they had no role in the conduct of the study.

\section{STATEMENT OF INTEREST}

None declared.

\section{ACKNOWLEDGEMENTS}

We would like thank N. Shang, M. Sternberg and J. Becerra for their statistical input, and P. Cegielski, A. Vernon and K. Castro (all National Center for HIV/AIDS, Viral Hepatits, STD and TB Prevention, US Centers for Disease Control and Prevention, Atlanta, GA, USA) for their insightful read of the manuscript.

\section{REFERENCES}

1 World Health Organization. Global Tuberculosis Control Report. Geneva, World Health Orgnization, 2009.

2 Centers for Disease Control and Prevention. Extensively drugresistant tuberculosis - United States, 1993-2006. MMWR 2007; 56: 250-253.

3 World Health Organization. Extensively drug-resistant tuberculosis (XDR-TB). Recommendations for prevention and control. Weekly Epidemiol Rec 2006; 81: 430-432.

4 Gandhi NR, Moll A, Sturm AW, et al. Extensively drug-resistant tuberculosis as a cause of death in patients co-infected with tuberculosis and HIV in a rural area of South Africa. Lancet 2006; 368: 1575-1580.

5 Holtz TH, Riekstina V, Zarovska E, et al. XDR TB: extensive drug resistance and treatment outcome under DOTS-Plus, Latvia, 20002002. Int J Tuberc Lung Dis 2005; 9: Suppl., S258.

6 Migliori GB, Ortmann J, Girardi E, et al. Extensively drug-resistant tuberculosis, Italy and Germany. Emerg Infect Dis 2007; 13: 780-782.

7 Migliori GB, Lange C, Girardi E, et al. Extensively drug-resistant tuberculosis is worse than multidrug-resistant tuberculosis: different methodology and settings, same results. Clin Infect Dis 2008; 46: 958-959.

8 Masjedi MR, Farnia P, Sorooch S, et al. Extensively drug-resistant tuberculosis: 2 years of surveillance in Iran. Clin Infect Dis 2006; 43: 841-847.

9 Lai CC, Tan CK, Huang YT, et al. Extensively drug-resistant Mycobacterium tuberculosis during a trend of decreasing drug resistance from 2000 through 2006 at a medical center in Taiwan. Clin Infect Dis 2008; 47: e57-e63.

10 Kwon YS, Kim YH, Suh GY, et al. Treatment outcomes for HIVuninfected patients with multidrug-resistant and extensively drug-resistant tuberculosis. Clin Infect Dis 2008; 47: 496-502.

11 Kim HR, Hwang SS, Kim HJ, et al. Impact of extensive drug resistance on treatment outcomes in non-HIV-infected patients with multidrug-resistant tuberculosis. Clin Infect Dis 2007; 45: 1290-1295. 
12 Migliori GB, Lange C, Centis R, et al. Resistance to second-line injectables and treatment outcomes in multidrug-resistant and extensively drug-resistant tuberculosis cases. Eur Respir J 2008; 31: 1155-1159.

13 Migliori GB, Besozzi G, Girardi E, et al. Clinical and operational value of the extensively drug-resistant tuberculosis definition. Eur Respir J 2007; 30: 623-626.

14 Sotgiu G, Ferrara G, Matteelli A, et al. Epidemiology and clinical management of XDR-TB: a systematic review by TBNET. Eur Respir J 2009; 33: 871-881.

15 Shah NS, Pratt R, Armstrong L, et al. Extensively drug-resistant tuberculosis in the United States, 1993-2007. JAMA 2008; 300: 2153-2160.

16 Kim DH, Kim HJ, Park SK, et al. Treatment outcomes and longterm survival in patients with extensively drug-resistant tuberculosis. Am J Respir Crit Care Med 2008; 178: 1075-1082.

17 Mitnick CD, Shin SS, Seung KJ, et al. Comprehensive treatment of extensively drug-resistant tuberculosis. N Engl J Med 2008; 359: 563-574.

18 Keshavjee S, Gelmanova IY, Farmer PE, et al. Treatment of extensively drug-resistant tuberculosis in Tomsk: Russia: a retrospective cohort study. Lancet 2008; 372: 1403-1409.

19 World Health Organization. Anti-tuberculosis Drug Resistance in the World: the WHO/IUATLD Global Project on Anti-Tuberculosis Drug Resistance Surveillance. Report No 4. Prevalence and Trends. Geneva, WHO, 2008.

20 Leimane V, Leimans J. Tuberculosis control in Latvia: integrated DOTS and DOTS-Plus programmes. Euro Surveill 2006; 11: 29-33.

21 Leimane V, Riekstina V, Holtz $\mathrm{TH}$, et al. Clinical outcome of individualised treatment of multidrug-resistant tuberculosis in Latvia: a retrospective cohort study. Lancet 2005; 365: 318-326.

22 Holtz TH, Riekstina V, Zarovska E, et al. Time to sputum culture conversion in multidrug-resistant tuberculosis: predictors and relationship to treatment outcome. Ann Intern Med 2006; 144: 650-660.

23 Riekstina V, Leimane V, Holtz TH, et al. Treatment outcome cohort analysis in an integrated DOTS and DOTS-Plus TB Program in Latvia. Int J Tuberc Lung Dis 2007; 11: 585-587.

24 Bonilla CA, Crossa A, Jave HO, et al. Management of extensively drug-resistant TB in Peru: cure is possible. PLoS One 2008; 3: e2957-e2963.

25 Kliiman K, Altraja A. Predictors of extensively drug-resistant pulmonary tuberculosis. Ann Intern Med 2009; 150: 766-775.

26 Centers for Disease Control and Prevention. Revised definition of extensively drug-resistant tuberculosis: notice to readers. MMWR 2006; 55: 1176.
27 World Health Organization. Guidelines for the Programmatic Management of Drug-Resistant Tuberculosis: Emergency Update 2008. Geneva, World Health Orgnization, 2008.

28 Reider HL, Chonde TM, Myking H, et al. The Public Health Service National Tuberculosis Reference Laboratory and the National Laboratory Network. Minimum Requirements, Role and Operation in a Low-Income Country. Paris, International Union Against Tuberculosis and Lung Disease, 1998.

29 Canetti G, Froman S, Grosset J, et al. Mycobacteria: laboratory methods for testing drug sensitivity and resistance. Bull WHO 1963; 29: 565-578.

30 Zignol M, Hosseini MS, Wright A, et al. Global incidence of multidrug-resistant tuberculosis. J Infect Dis 2006; 194: 479-485.

31 Nardell EA, Mitnick CD. Are second-line drugs necessary to control multidrug-resistant tuberculosis? J Infect Dis 2006; 194: 1194-1196.

32 Skenders G, Holtz TH, Toma A, et al. Introducing an assay for rapid detection of rifampin resistance into clinical care of MDR-TB patients in Latvia. Int J Tuberc Lung Dis 2007; 1: Suppl. 1, S81.

33 Caminero JA. Extensively drug-resistant tuberculosis: is its definition correct? Eur Resp J 2008; 32: 1413-1415.

34 Chiang C-Y, Enarson DA, Yu M-C, et al. Outcome of pulmonary multidrug-resistant tuberculosis: a 6-yr follow-up study. Eur Respir J 2006; 28: 980-985.

35 Johansen IS, Larsen AR, Sandven P, et al. Drug susceptibility testing of Mycobacterium tuberculosis to fluoroquinolones: first experience with a quality control panel in the Nordic-Baltic collaboration. Int J Tuberc Lung Dis 2003; 7: 899-902.

36 World Health Organization. Policy Guidance on DrugSusceptibility Testing of Second-Line Antituberculosis Drugs. Geneva, World Health Orgnization, 2008.

37 National Committee on clinical Laboratory Standards. Susceptibility Testing of Mycobacteria, Nocardiae, and Other Aerobic Actinomycetes: Approved Standard. Wayne, National Committee on clinical Laboratory Standards, 2003.

38 World Health Organization. Report of the Second Meeting of the WHO Task Force on XDR-TB. Geneva, WHO, 2008.

39 Devaux I, Kremer K, Heersma H, et al. Clusters of multidrugresistant Mycobacterium tuberculosis cases, Europe. Emerg Infect Dis 2009; 15: 1052-1060.

40 Kim SJ, Espinal MA, Abe C, et al. Is second-line anti-tuberculosis drug susceptibility testing reliable? Int J Tuberc Lung Dis 2004; 8: 1157-1158.

41 Ginsburg AS, Grosset JH, Bishai WR. Fluoroquinolones, tuberculosis, and resistance. Lancet Infect Dis 2003; 3: 432-442.

42 Iseman MD, Heifets LB. Rapid detection of tuberculosis and drugresistant tuberculosis. New Engl J Med 2006; 355: 1606-1608. 